\title{
Transmural colonic migration of a retained surgical sponge (Gossypiboma) which clinically mimicked a colonic tumor: A case report
}

\author{
Ramón Vidrio-Duarte ${ }^{1 *}$, Elliot Correa-Dip ${ }^{1}$, Eduardo Vidrio-Duarte ${ }^{2}$, Hugo I. Aguilar-Preciado ${ }^{1}$, \\ Juan A. Gutierrez-Ochoa ${ }^{1}$, and Agustin Chávez-Gómez ${ }^{1}$ \\ ${ }^{1}$ Department of Surgery, Hospital General de México "Dr. Eduardo Liceaga"; ${ }^{2}$ Bariatric Surgery, Hospital Ángeles Metropolitano. Mexico City, Mexico
}

\begin{abstract}
We report the case of a 30-year-old female being studied for a colonic tumor, on the computed tomography-scan an abdominal mass with wall reinforcement and air bubbles suggestive of a retained sponge, Barium enema showed an extraluminal radiopaque marker and a mass migrated into the colon, on surgery, an enterocolic fistula was found, and we resected the ascending colon and $40 \mathrm{~cm}$ of terminal ileum and performed a ileotranverse anastomosis. Gossypibomas, despite preventive measures, remain a concern for surgeons due to its medicolegal implications, a high clinical suspicion leads to a correct diagnostic protocol and an early treatment.
\end{abstract}

Key words: Gossypiboma. Surgical sponge. Textiloma. Foreign bodies. Fistula.

\section{Introduction}

The term "Gossypiboma", also reported in the literature as textiloma, cottonoid, cottonballoma, muslinomas, and gauzeoma, has two possible origins, some authors believe that it is derived from the gossip among surgeons that this entity could entail ${ }^{1}$, on the other hand, the most accepted origin relies on the etymology of the word, "Gossypium" which comes from "gossypinus" that in Latin means cotton plant, and "boma" meaning in Swahili enclosure or fortified outpost $^{2}$. The first case was reported in 1884 by Wilson; since this first published case, many authors have reported their experience mainly on case reports and just a few series ${ }^{3}$. The exact incidence of gossypibomas is unknown, it is estimated from $0.01 \%$ to $0.001 \%{ }^{4}$, it has a low reporting rate due to medicolegal implications, fear of litigation that could lead to high expenses and compensations, and also adverse publicity not only for surgeons but also for medical institutions ${ }^{5}$; despite preventive protocols such as standardized surgical count and the use of radiography when the surgical count is incorrect this entity still appears as a complication of nowadays' procedures ${ }^{6}$. The wide diversity of clinical manifestations makes it difficult to diagnose; thus, a high suspicion based on the surgical history of the patient is needed; the most common symptoms are abdominal pain $(73 \%)$ and a palpable mass $(47 \%)^{7}$.

\section{Case Presentation}

A 30-year-old female presented to the Outpatient Department of Surgery at the Hospital General de México "Dr. Eduardo Liceaga" with a long-standing vague colicky abdominal pain, without a triggering cause and

\section{Correspondence:}

*Ramón Vidrio-Duarte

E-mail: ramon.rvd@gmail.com
Available online: 12-11-2020

Date of reception: 29-07-2019

Date of acceptance: 08-04-2020 DOI: $10.24875 /$ HGMX.20000052
Rev Med Hosp Gen Mex. 2020;83(4):168-171

www.hospitalgeneral.mx NC-ND license (http://creativecommons.org/licenses/by-nc-nd/4.0/). 
associated with nausea, no weight loss or intestinal bleeding was referred. The patient also referred asymptomatic anemia after a scheduled cesarean section performed 20 months prior to her current admission in another hospital, supposedly with no complications or major bleeding. At first, the patient was directed to the oncology department due to an external abdominal ultrasound which found an intra-abdominal non-specified tumor. Tumoral markers were negative and the computed tomography (CT)-scan ruled out neoplasia. On physical examination, the patient had good general condition, a bloated abdomen with a midline infraumbilical scar, and a non-tender $10 \times 6 \mathrm{~cm}$ slightly mobile mass in the right flank and right hypochondrium.

CT-scan showed a heterogeneous mass with peripheral contrast enhancement, hypodense material, and fine hyperdense lines, along with air bubbles within ascending colon and hepatic flexure that allows oral contrast flow, associated with colon wall enlargement and suggestive images of intestinal pneumatosis (Fig. 1). In addition, a Barium enema was performed and demonstrated on the initial projection a radiopaque marker localized in mesogastrium, with contrast administration, we observe an intracolonic mass with slight calcifications localized in the mid-transverse colon, right colic flexure, and down to the mid-ascending coIon, and no flow of contrast to the cecum, descending colon seems to be pulled up by this mass (Fig. 2).

Consequently, a scheduled exploratory laparotomy was performed, in the abdominal cavity, a $15 \times 10$ firm mass was found which on the inside contained a textile, fetid, and greenish material which migrated into the colon and generated an enterocolic fistula of at least 6 $\mathrm{cm}$ wide, ascending colon and $40 \mathrm{~cm}$ of distal ileum where resected and a manual ileotransverse anastomosis was performed, the total blood loss on the procedure was $400 \mathrm{ml}$. (Fig. 3). The patient had a favorable outcome, the diet was introduced on the $3^{\text {rd }}$ post-operative day, and she was discharged on the $7^{\text {th }}$ post-operative day, the patient has been asymptomatic and clinically stable for 12 months.

\section{Discussion}

Some studies with heterogeneous methodology had identified several risk factors, leading to a retained material. Lincourt et al. identified the number of procedures performed during the same intervention and incorrect material count as risk factors; another study by Gawande et al., the authors found emergency surgeries, a high body mass index, and an unexpected change in

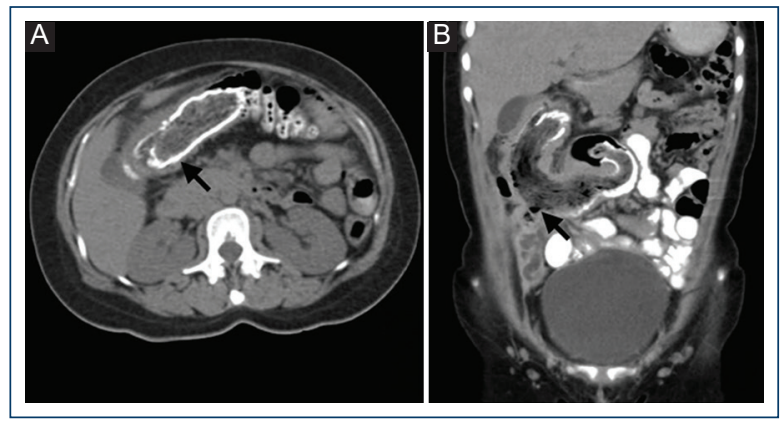

Figure 1. CT-scan showing pathognomonic findings of a Gossypiboma. A: arrow showing enhancement of the capsule. B: arrow showing air bubbles.

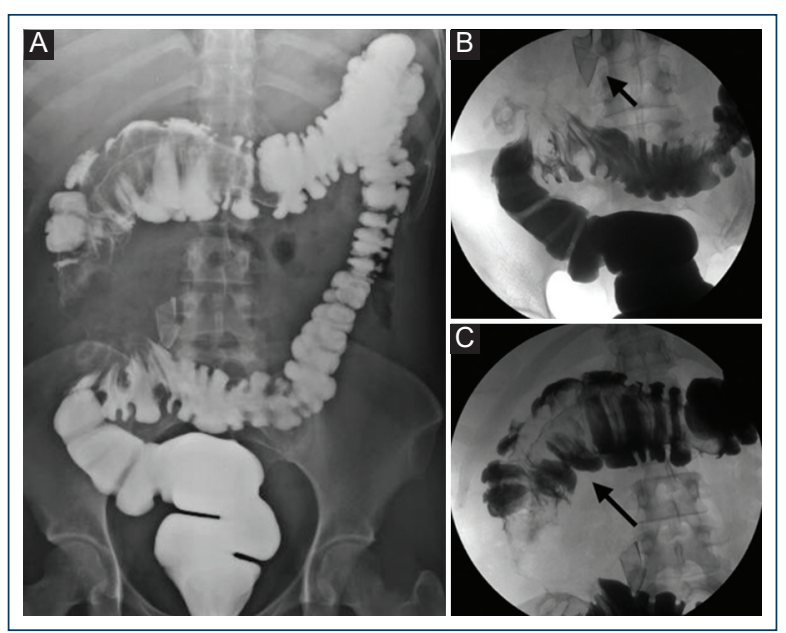

Figure 2. Barium Enema. A: abdominal involving the ascending colon. B: arrow; extraluminal radiopaque marker; affection with a pulled up appearance of the descendind colon. C: arrow; intraluminal mass on the right flexure and transverse colon.

operation as the mayor risk factors ${ }^{8,9}$. In addition, in a systematic review by Patial et al., the authors identified on the patients diagnosed with a gossypiboma, a higher incidence when a gynecologic $(41.2 \%)$ or abdominal surgery $(35.7 \%)$ were performed ${ }^{7}$, this correlates with other reports ${ }^{1,10,11}$. Hence, many institutions emphasize on surgical protocols to reduce this risk, there is a high value on counting materials before and after its use and never start closure with an incomplete count as stated in the WHO Surgery Checklist Implementation Manu$\mathrm{al}^{12,13}$. Increased duration of surgery, a shift of the nursing team, emergency surgery, and surgical procedures on the night shift are the main causes of an incomplete count ${ }^{14}$. In addition, in a retrospective review published in 2017, the authors highlight the importance of 


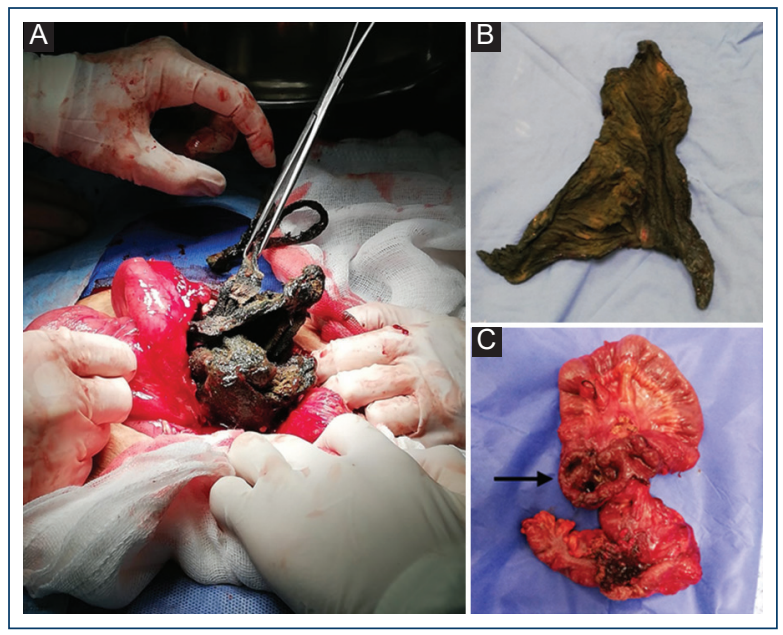

Figure 3. intraoperative findings. A: surgical sponge being pulled out of the intestinal lumen. B: retained surgical sponge $30 \times 30 \mathrm{~cm}$. C: arrow; enterocolic fistula.

radiofrequency detection as new technological options to reduce the risk, on a previous study by the same group, they found a $100 \%$ sensitivity identifying retained sponges when low frequency radiofrequency was used ${ }^{15}$. They also point out that this new technology has better sensitivity than radiographs associated with the use of radiopaque markers, mainly considering that this is not a routine procedure, and they are only used when the sponge count is incorrect ${ }^{11,16}$. Nevertheless, special attention on the surgical count reduces the risk of foreign material retention, especially in surgical centers where these technologies are not available $6,17,18$.

In regard to pathogenesis, it is important to highlight the two types of body responses, this body reacting could be either fibrinous or exudative. With low antigenicity, a mild inflammatory response occurs developing an aseptic fibrinous response, causing encapsulation of the material, adhesion formation, and calcifications. The exudative with high antigenicity develops a rapid, severe inflammation, leading to abscess formation and or fistula and even migration into the intestinal tract ${ }^{19}$; once inside the lumen, its migration is favored by peristalsis; subsequently, the sponge could be eliminated with feces or cause obstruction of the intestinal lu$\operatorname{men}^{7,20,21}$. Notably, image studies play a crucial role in diagnosis, there are two scenarios, on the first one, it presents as an incidental finding; on the other hand, there are patients with a history of a surgical procedure and who afterward develops suggestive symptoms; consequently, the attendant requests an image study to confirm his diagnosis 7,10 . Nowadays, CT scan is the imaging technique of choice, it shows a low density and well defined heterogeneous mass with capsule reinforcement and air bubbles inside or even barium if there is migration into the intestinal lumen; the radiopaque marker appears as a narrow metallic line within the mass, the "calcified reticulated rind sing" develops on long-lasting sponges ${ }^{22}$. Likewise, an MRI shows a gossypiboma as a low-density mass with thick wall and a whorled internal configuration on T2-weighted imaging; however, this study is incapable of visualizing the radiopaque marker. Even though these image techniques are more specific, radiographs are still the most commonly performed study; nonetheless, this modality gives no information with regard to complications. Notably, a barium enema is infrequently performed; in our case, this study gave us plenty of information not only in relation to diagnosis but also for surgical planning; consequently, we recommend barium enema, especially in centers where CT-scan is not available 2,11,21,23,24.

With regard to the medicolegal implications that a gossypiboma carries, these differ amongst countries, but the essence of the issue has been found to concur on different legal systems; in some cases, gossypibomas lead to claims of medical malpractice and in others could lead to professional suspension, monetary compensation or even worse consequences; in this cases, it is relevant to determine whether a complete count of material was reported or not, since the first scenario may partially absolve the surgeon, other parameters that could diminish the operating team's responsibility are when several sponges were used in mayor bleeding or trauma surgery, also when there was a miscount reported, but the hemodynamic status of the patient is critical, and there is no time for further pursuit of the missing sponge. Nevertheless, a retained foreign body after surgery remains in the majority of cases as an inexcusable fault, especially in the context of an elective procedure. In our country, there is few literature in relation to medicolegal outcomes of negligence claims ${ }^{5,25}$.

Ultimately, the surgical team has to pay especial attention to the count of surgical materials before and during surgery, and additionally, apply "time out" protocols before closure of the abdominal wall to reduce the risk of missing sponges; the surgeon must be aware of this risk and take responsibility of double-checking protocols, rather than delegate it only to the nursery team.

\section{Conclusion}

Despite preventive measures, Gossypibomas remain a concern for surgeons, not only due to its medicolegal 
implications but also for its undesirable morbidity and mortality; a high clinical suspicion leads to a correct diagnostic protocol and an early treatment.

The preventive measures of adhesion to safety protocols lead to a lower incidence of this pathology; the entire surgical team must adopt these preventive actions to benefit the patients.

\section{Funding}

The authors did not receive any kind of financial support.

\section{Conflicts of interest}

The authors declare that they have no conflicts of interest.

\section{Ethical disclosures}

Protection of human and animal subjects. The authors declare that no experiments were performed on humans or animals for this study.

Confidentiality of data. The authors declare that they have followed the protocols of their work center on the publication of patient data.

Right to privacy and informed consent. Right to privacy and informed consent. The authors have obtained the written informed consent of the patients or subjects mentioned in the article. The corresponding author is in possession of this document.

\section{References}

1. Bani-Hani K, Gharaibeh K, Yagha R. Retained surgical sponges (gossypiboma). Asian J Surg. 2005;28:109-15.

2. Ogundiran T, Ayandipo O, Adeniji-Sofoluwe A, Ogun G, Oyewole O Ademola A. Gossypiboma: complete transmural migration of retained surgical sponge causing small bowel obstruction. BMJ Case Rep. 2011;2011:4073.

3. Wilson C. Foreign bodies left in the abdomen after laparotomy. Trans Am Gynecol Soc. 1884:9:94-117.

4. Kim HS, Chung TS, Suh SH, Kim SY. MR imaging findings of paravertebral gossypiboma. AJNR Am J Neuroradiol. 2007;28:9-13.

5. Garg M, Aggarwal AD. A review of medicolegal consequences of gossypiboma. J Indian Acad Forensic Med. 2010;32:358-61.

6. Hariharan D, Lobo D. Retained surgical sponges, needles and instruments. Ann R Coll Surg Engl. 2013;95:87-92.

7. Patial T, Thakur V, Ganesun NV, Sharma M. Gossypibomas in India-a systematic literature review. J Postgrad Med. 2017;63:36.

8. Lincourt A, Harrell A, Cristiano J, Sechrist C, Kercher K, Heniford B. Retained foreign bodies after surgery. J Surg Res. 2007;138:170-4.

9. Gawande A, Studdert D, Orav E, Brennan T, Zinner M. Risk factors for retained instruments and sponges after surgery. $\mathrm{N}$ Engl $\mathrm{J}$ Med. 2003;348:229-35.

10. Arikan S, Kocakusak A. Retained textile foreign bodies: experience of 27 years. Acta Méd Por. 2015;28:494.

11. Steelman V, Shaw C, Shine L, Hardy-Fairbanks A. Retained surgical sponges: a descriptive study of 319 occurrences and contributing factors from 2012 to 2017. Patient Saf Surg. 2018;12:20.

12. World Alliance for Patient Safety. Implementation Manual: WHO Surgical Safety Checklist. $1^{\text {st }}$ ed. Geneva, Switzerland: Safe Surgery Safe Live, World Health Organization; 2008.

13. World Alliance for Patient Safety. WHO Guidelines for Safe Surgery. Geneva, Switzerland: World Health Organization; 2009.

14. Egorova NN, Moskowitz A, Gelijns A, Weinberg A, Curty J, Rabin-Fastman $B$, et al. Managing the prevention of retained surgical instruments: what is the value of counting? Ann Surg. 2008;247:13-8.

15. Steelman V. Sensitivity of detection of radiofrequency surgical sponges: a prospective, cross-over study. Am J Surg. 2011;201:233-7.

16. Williams T, Tung D, Steelman V, Chang P, Szekendi M. Retained surgical sponges: findings from incident reports and a cost-benefit analysis of radiofrequency technology. J Am Coll Surg. 2014;219:354-64.

17. Freitas P, Silveira R, Clark A, Galvão C. Surgical count process for prevention of retained surgical items: an integrative review. J Clin Nurs. 2016;25:1835-47.

18. Fencl J. Guideline implementation: prevention of retained surgical items. AORN J. 2016;104:37-48.

19. Agrawal H, Gupta N, Krishengowda U, Gupta A, Naskar D, Durga C. Transmural migration of gossypiboma: a rare cause of acute abdomen. Indian J Surg. 2017;80:84-6.

20. Kassi A, Yenon K, Koffi E. A transmural migration of a gossypiboma in the right colon responsible for a mass which mimicked an abscessed colonic tumor: a case report. Int J Surg Case Rep. 2018;51:228-30.

21. Dux M, Ganten M, Lubienski A. Retained surgical sponge with migration into the duodenum and persistent duodenal fistula. Eur Radiol. 2002;12:S74-7.

22. Lu YY, Cheung YC, Ko SF, Ng SH. Calcified reticulate rind sign: a characteristic feature of gossypiboma on computed tomography. World $\mathrm{J}$ Gastroenterol. 2005;11:4927.

23. Manzella A, Filho P, Albuquerque E, Farias F, Kaercher J. Imaging of gossypibomas: pictorial review. Am J Roentgenol. 2009;193:S94-101.

24. Butt $U$, Shafiq A, Umar M, Ashfaq M, Ayyaz M. Transmigration and spontaneous passage of a gossypiboma documented on contrast study. Ann Med Surg. 2019;38:42-4.

25. Gualniera $P$, Scurria $S$. Retained surgical sponge: medicolegal aspects. Legal Med. 2018;31:78-81. 